\title{
Calculations of $\bar{K}$-nuclear quasi-bound states using chiral $\bar{K} N$ amplitudes
}

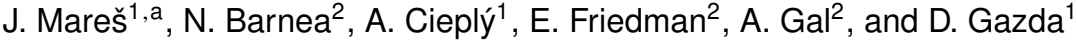 \\ ${ }^{1}$ Nuclear Physics Institute, 25068 Řež, Czech Republic \\ ${ }^{2}$ Racah Institute of Physics, The Hebrew University, 91904 Jerusalem, Israel
}

\begin{abstract}
We review our recent calculations of $K^{-}$quasi-bound states in nuclear systems using subthreshold energy dependent chiral $\bar{K} N$ amplitudes. Strong energy dependence of the scattering amplitudes requires self-consistent evaluation of the involved $\bar{K} N$ interactions. In view of sizable widths predicted by our calculations, an unambiguous identification of $\mathrm{K}^{-}$-nuclear quasi-bound states in ongoing experimental searches would be difficult.
\end{abstract}

\section{Introduction}

Most of the current calculations of $K^{-}$-nuclear quasi-bound states are based on the $\bar{K} N$ interactions derived within an $\mathrm{SU}(3)$ chiral approach combined with coupled channel T-matrix resummation techniques [1-3]. In such an approach, constrained by fitting to $K^{-} p$ threshold and low-energy data, the $\Lambda(1405)$ resonance is generated dynamically. Its presence induces strong energy dependence in the scattering amplitudes $f_{\bar{K} N}(\sqrt{s})$ which has to be treated self-consistently, as shown in refs. [4-6]. The key issue is that in the nuclear medium (for $A \gg 1$ approximated by the lab system)

$$
\sqrt{s}=\sqrt{\left(\sqrt{s_{\mathrm{th}}}-B_{K}-B_{N}\right)^{2}-\left(\vec{p}_{K}+\vec{p}_{N}\right)^{2}} \leq \sqrt{s_{\mathrm{th}}}
$$

where $\sqrt{s_{\mathrm{th}}} \equiv m_{K}+m_{N}$ and $B_{K}\left(B_{N}\right)$ is the antikaon (nucleon) binding energy and the momentum dependent term generates additional substantial downward energy shift, since $\left(\vec{p}_{K}+\vec{p}_{N}\right)^{2} \neq 0$, unlike the case of the two-body $\mathrm{cm}$ system.

Main results of our recent calculations of $K^{-}$quasi-bound states in nuclear few-body [6] and many-body $[4,5]$ systems are summarized in the following sections.

\section{Few-body kaonic nuclear clusters}

We performed calculations of three-body $(\bar{K} N N)_{I=0}$ and four-body $(\bar{K} N N N)_{I=0,1}$ and $(\bar{K} \bar{K} N N)_{I=0}$ nuclear quasi-bound states. The $\bar{K}$-nuclear cluster wavefunctions were expanded in a hyperspherical

\footnotetext{
ae-mail: mares@ujf.cas.cz
}

This is an Open Access article distributed under the terms of the Creative Commons Attribution License 2.0, which permits unrestricted use, distribution, and reproduction in any medium, provided the original work is properly cited. 
basis and the ground-state binding energies were calculated variationally. The corresponding $\bar{K} N \rightarrow$ $\pi Y$ widths were evaluated using the expression:

$$
\frac{\Gamma}{2} \approx\left\langle\Psi_{\mathrm{g} . s .}\left|-\operatorname{I} m V_{\bar{K} N}\right| \Psi_{\mathrm{g} . s .}\right\rangle
$$

where $V_{\bar{K} N}$ sums overall pairwise $\bar{K} N$ interactions. For two-body interactions involved, we used the AV4' $V_{N N}$ [7], an effective energy-dependent $V_{\bar{K} N}$ [8] and a weakly repulsive $V_{\bar{K} \bar{K}}$ [9]. In $\bar{K}$ nuclear clusters, the energy dependent $V_{\bar{K} N}(\sqrt{s})$ was evaluated self-consistently for $\sqrt{s}$, expressed near threshold in the form:

$$
\sqrt{s}=\sqrt{s_{\mathrm{th}}}-\frac{B}{A}-\frac{A-1}{A} B_{K}-\xi_{N} \frac{A-1}{A}\left\langle T_{N N}\right\rangle-\xi_{K}\left(\frac{A-1}{A}\right)^{2}\left\langle T_{K}\right\rangle,
$$

where $\xi_{N(K)}=m_{N}(K) /\left(m_{N}+m_{K}\right), B$ is the total binding energy of the system, $T_{K}$ is the kaon kinetic energy operator in the total $\mathrm{cm}$ frame and $T_{N N}$ is the pairwise $N N$ kinetic energy operator in the $N N$ pair cm system. A similar procedure was used for the $\bar{K} \bar{K} N N$ cluster (see [6] for details).

Results of our self-consistent calculations are summarized in Fig. 1. Since $\bar{K} N$ amplitudes (and consequently potentials) decrease upon going subthreshold, self-consistent calculations yield binding energies and widths of the calculated nuclear clusters lower than calculations performed at threshold, typically $\Delta B \sim 10 \mathrm{MeV}$ and $\Delta \Gamma \sim 10-40 \mathrm{MeV}$. We reproduced results of previous $\bar{K} N N_{I=0}$ calculations using chiral energy-dependent $\bar{K} N$ amplitudes [10]. In view of the low $\bar{K} N N_{I=0}$ binding energy $B(\bar{K} N N) \approx 16 \mathrm{MeV}$ and relatively large absorption width $\Gamma(\bar{K} N N) \approx 40 \mathrm{MeV}$, it might be difficult to identify the $K^{-} p p$ quasi-bound state unambiguously in ongoing experiments.

We found relatively modest binding of the four-body $\bar{K}$ nuclear clusters, about $30 \mathrm{MeV}$ in the lowest $I=0$ systems, with absorption widths ranging from $30 \mathrm{MeV}$ for $\bar{K} N N N$ to about $80 \mathrm{MeV}$ for the $\bar{K} \bar{K} N N$ quasi-bound state.

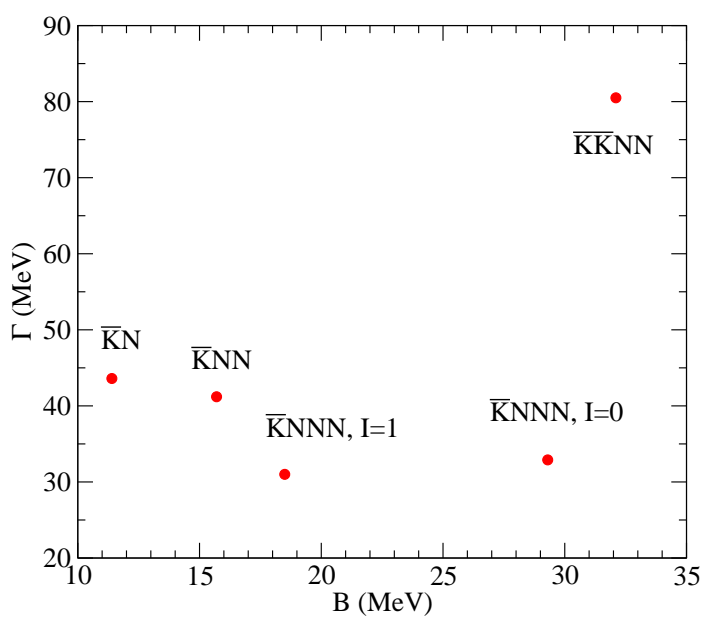

Figure 1. Calculated binding energies and $\bar{K} N \rightarrow \pi Y$ widths of few-body $\bar{K}$-nuclear clusters [6]. 
It is to be noted that the widths shown in Fig. 1 are due to $\bar{K} N \rightarrow \pi Y$. Two-nucleon $K^{-} N N \rightarrow Y N$ absorption widths are expected to add $\Delta \Gamma \lesssim 10 \mathrm{MeV}$ in $\bar{K} N N_{I=0}$ system [10] and $\sim 20 \mathrm{MeV}$ in the 4-body clusters [6]. The binding energies of $K^{-}$nuclear clusters could also be enhanced by dispersive contributions. Our recent fits to kaonic atoms [4, 11] suggest that $\Delta B_{\text {disp }} \sim \Delta \Gamma_{\text {abs }}$, and the binding energies could reach values $B\left(K^{-} p p\right) \sim 25 \mathrm{MeV}$ and $B(\bar{K} N N N, \bar{K} \bar{K} N N) \sim 50 \mathrm{MeV}$.

\section{Many-body $K^{-}$-nuclear systems}

The $K^{-}$-nuclear quasi-bound states were calculated within the RMF formalism (see Refs. [4, 5] for details). The interaction of a $K^{-}$meson with a nucleus is described by the Klein-Gordon (KG) equation of the form

$$
\left[\nabla^{2}+\tilde{\omega}_{K}^{2}-m_{K}^{2}-\Pi_{K}\left(\omega_{K}, \vec{p}_{K}, \rho\right)\right] \phi=0,
$$

where $\tilde{\omega}_{K}=\omega_{K}-\mathrm{i} \Gamma_{K} / 2-V_{C}$ is complex energy of antikaon containing the Coulomb interaction $V_{C}$, with $\Gamma_{K}$ being the width of $K^{-}$nuclear state of energy $\omega_{K}=m_{K}-B_{K}$. The self-energy operator $\Pi_{K}=2\left(\operatorname{Re} \omega_{K}\right) V_{K}$ reads:

$$
\Pi_{K}\left(\omega_{K}, \vec{p}_{K}, \rho\right)=-4 \pi \frac{\sqrt{s}}{m_{N}}\left[F_{K^{-} p}(\sqrt{s}, \vec{p}, \rho) \rho_{p}+F_{K^{-} n}(\sqrt{s}, \vec{p}, \rho) \rho_{n}\right],
$$

where $F_{K^{-} p(n)}$ is the $K^{-}$-proton (neutron) in-medium scattering amplitude constructed within a chirally motivated coupled-channel model and $\rho_{p}\left(\rho_{n}\right)$ is the proton (neutron) RMF density distribution in a core nucleus. The scattering amplitudes in Eq. (5) are a function of $\bar{K} N$ c.m. energy $\sqrt{s}$ and relative momentum $\vec{p}$. The transformation of the two-body $\bar{K} N$ arguments into the $\bar{K}$-nuclear c.m. frame (for $A \gg 1$ ) leads to:

$$
\begin{array}{ll}
p^{2} \approx \xi_{N} \xi_{K}\left[2 m_{K} 23\left(\rho / \rho_{0}\right)^{2 / 3}-2 m_{N}\left(B_{K}+\operatorname{Re} \mathcal{V}_{K}(\rho)\right)\right] & (\text { in } \mathrm{MeV}), \\
\sqrt{s} \approx \sqrt{s_{\mathrm{th}}}-B_{N}-\xi_{N} B_{K}-15.1\left(\frac{\rho}{\rho_{0}}\right)^{2 / 3}+\xi_{K} \operatorname{Re} \mathcal{V}_{K}(\rho) & (\text { in MeV }),
\end{array}
$$

where $\mathcal{V}_{K}=V_{K}+V_{C}$.

We note that the $K^{-}$potential $V_{K}$ and the $K^{-}$binding energy $B_{K}$ appear as arguments in the expression for $\sqrt{s}$, which in turn serves as an argument for the self energy $\Pi_{K}$, and thus for $V_{K}$. Therefore, a self-consistency scheme in terms of both $V_{K}$ and $B_{K}$ is required for solving the KG equation (4).

We considered two in-medium versions of the scattering amplitudes: the version which takes into account only Pauli blocking in the intermediate states, and the version $(+\mathrm{SE})$ which adds selfconsistently hadron in-medium self-energies $[4,5]$. The $\bar{K} N$ amplitudes were constructed using the in-medium coupled-channel separable interaction model NLO30 [3] that reproduces all available low energy $\bar{K} N$ observables, including the latest $1 s$ level shift and width in the $K^{-}$hydrogen atom from the SIDDHARTA experiment [12]. While the two in-medium versions of the $\bar{K} N$ scattering amplitudes yield by factor 2 different potential depths $\operatorname{Re} V_{K}$ at threshold, they give similar depths in the selfconsistent calculations with the subthreshold extrapolation, $\operatorname{Re} V_{K} \sim 80-120 \mathrm{MeV}$, depending on a particular nucleus.

Figure 2 shows binding energies and widths of $\mathrm{K}^{-}$quasi-bound states - including excited states - in selected nuclei calculated self-consistently for $\sqrt{s}$ of Eq. (7), using the '+SE' amplitudes. The widths of low-lying $K^{-}$states due to $K^{-} N \rightarrow \pi Y$ conversions are substantially reduced in the selfconsistent calculations, thus reflecting the proximity of the $\pi \Sigma$ threshold. On the contrary, the widths of higher excited $K^{-}$states are quite large even if only the pion conversion modes on a single nucleon are considered. After including 2 body $K^{-} N N \rightarrow Y N$ absorption modes, the total decay widths $\Gamma_{K}$ are 

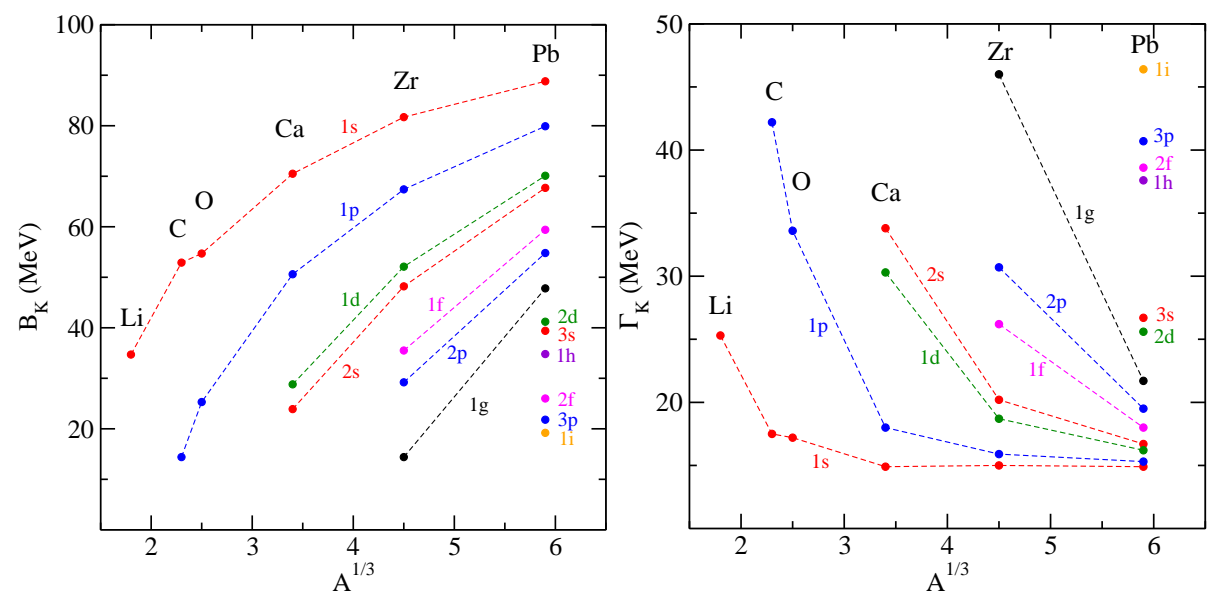

Figure 2. Binding energies $B_{K}$ (left panel) and widths $\Gamma_{K}$ (right panel) of $K^{-}$quasi-bound states in selected nuclei, calculated self-consistently with the '+SE' NLO30 scattering amplitudes [3]. $K^{-} N N \rightarrow Y N$ absorption modes are not included.

comparable or even larger than the corresponding binding energies $B_{K}$ for all $K^{-}$nuclear quasi-bound states, exceeding considerably the level spacing. Our results should thus discourage attempts to search for isolated peaks corresponding to $K^{-}$nuclear quasi-bound states in many-body nuclear systems.

\section{Acknowledgments}

This work was supported by the GACR grant No. 203/12/2126 and by the EU inciative FP7, HadronPhysics3 (SPHERE and LEANNIS networks).

\section{References}

[1] A. Ramos, E. Oset, Nucl. Phys. A 671, 481 (2000)

[2] B. Borasoy, R. Nissler, W. Weise, Eur. Phys. J. A 25, 79 (2005); Phys. Rev. Lett. 94, 213401 (2005)

[3] A. Cieplý, J. Smejkal, Nucl. Phys. A 881, 115 (2012)

[4] A. Cieplý, E. Friedman, A. Gal, D. Gazda, J. Mareš, Phys. Lett. B 702, 402 (2011); Phys. Rev. C 84, 045206 (2011)

[5] D. Gazda, J. Mareš, Nucl. Phys. A 881, 159 (2012)

[6] N. Barnea, A. Gal, E. Z. Liverts, Phys. Lett. B 712, 132 (2012)

[7] R. B. Wiringa, S. C. Pieper, Phys. Rev. Lett. 89, 182501 (2002)

[8] T. Hyodo, W. Weise, Phys. Rev. C 77, 035204 (2008)

[9] Y. Kanada-En'yo, D. Jido, Phys. Rev. C 78, 025212 (2008)

[10] A. Doté, T. Hyodo, W. Weise, Nucl. Phys. A 804, 197 (2008); Phys. Rev. C 79, 014003 (2009)

[11] E. Friedman, A. Gal, Nucl. Phys. A 881, 150 (2012); Nucl. Phys. A 899, 60 (2013)

[12] M. Bazzi et al. (SIDDHARTA Collaboration), Phys. Lett. B 704, 113 (2011); Nucl. Phys. A 881, 88 (2012) 